\title{
DISEÑO DE UN MODELO DE ESTIMACIÓN DE COSTOS EFICIENTES PARA EXPLOTACIONES GANADERAS DE PEQUEÑOS PRODUCTORES CRIANCEROS UBICADOS ENTRE LAS REGIONES DEL MAULE Y DE LOS LAGOS
}

\author{
DESIGNING OF AN EFFICIENT EVALUATION COST MODEL \\ FOR THE STOCKBREEDER EXPLOITATION OF THE SMALL PRODUCERS \\ FROM THE VII TO THE X REGIONS
}

\author{
Luis SÁez Tonaccaํ, Gloria Bucarey Oyanedel², Michel LePorati NeróN³
}

\begin{abstract}
The sanitary and commercial advantages of Chile have given the only opportunity for the development of stockbreeding bovine, transforming it in a new opportunity is strongly presented for the small breeding producers of our country, since that they are who initiate the productive cycle of bovine meat. The challenge for them is based on increasing their stock breeder mass, in volume and quality, for this reason, they obligatory need to be more efficient in their costs. The objective of this investigation was the designing of an efficient evaluation cost model for the stock breeder exploitation of the small producers of our country. The real productive costs, associated to the system of breeding, were determined through a survey applied to the small stockbreeder producers from the VII to the X regions. Besides, the relevant variables that influence in cost of production of system of breeding and mathematics expressions used for quantify these variables, were determined. For determining the better productive practices it was applied the technique of data evolvement analysis, by means of which the efficient producers were identified. The model of evaluation costs was based on the concept of comparative efficiency or benchmarking, by means of which, an efficient system of breeding that incorporated the best productive practices and technical characteristics of the most efficient producers, was designed.
\end{abstract}

KEY WORDS: Benchmarking, data evolvement analysis.

\section{RESUMEN}

\begin{abstract}
Las ventajas de tipo sanitario y comercial de Chile han dado una oportunidad única para el desarrollo de la ganadería bovina, transformándola en un nuevo polo exportador. Esta nueva oportunidad se presenta fuertemente para los pequeños productores crianceros de nuestro país, ya que son ellos los que inician el ciclo productivo de la carne bovina. El desafio para ellos está en aumentar su masa ganadera en volumen y calidad para lo cual necesitan obligatoriamente ser cada vez más eficientes en sus costos. El objetivo de esta investigación fue diseñar un modelo de estimación de costos eficientes para explotaciones ganaderas de los pequeños productores crianceros de nuestro país. Se determinaron los costos productivos reales asociados al sistema de crianza a través de una encuesta que se aplicó a pequeños productores crianceros desde la VII a la X regiones. Se determinaron además las variables relevantes que influyen en los costos de producción del sistema de crianza y las expresiones matemáticas utilizadas para cuantificar estas variables. Para determinar las mejores prácticas productivas se aplicó la técnica del Análisis Envolvente de Datos (DEA), con la cual se identificaron los productores eficientes. El modelo de estimación de costos se basó en el concepto de eficiencia comparativa o benchmarking, por lo que se diseñó un sistema de crianza modelo o eficiente que incorpora las mejores prácticas productivas y las características técnicas de los productores más eficientes.
\end{abstract}

PALABRAS ClaVE: Eficiencia comparativa, análisis envolvente de datos.

\footnotetext{
Universidad de Santiago de Chile; luissaez@usach.cl

Universidad de Santiago de Chile; gloria_negocios@yahoo.es

Ministerio de Agricultura; mleporati@minagri.gob.cl
} 


\section{INTRODUCCIÓN}

La industria de la carne bovina en Chile es un sector pequeño en producción, presenta una reducida masa ganadera y ha sido uno de los sectores más afectados por las importaciones en este último tiempo (Catrileo, 2005).

La crisis de precios del sector bovino en Chile surge desde que se liberan las trabas arancelarias y para-arancelarias existentes para las importaciones de carne a mediados de la década de los 70. Desde entonces, los precios de la carne en Chile comienzan a ser afectados por los precios de gigantes de la actividad ganadera mundial como son Argentina, Paraguay, Uruguay y Brasil. Estos países, con innumerables ventajas comparativas para producir carne respecto de nuestro país, provocan una aguda crisis de rentabilidad del negocio, ya que a partir de entonces los precios del ganado en Chile comienzan a ser fijados en referencia a precios del MERCOSUR (Catrileo, 2005).

Con seguridad los más afectados por este nuevo escenario han sido los pequeños productores crianceros, $\mathrm{y}$ a que son ellos los que inician el ciclo productivo de la carne bovina y cuya principal motivación para mantenerse en el negocio es la autosubsistencia de sus economías familiares.

Los pequeños productores crianceros producen terneros desde su nacimiento hasta su destete, alrededor de los 6-7 meses de edad, donde los animales cuentan con un peso aproximado de $220 \mathrm{~kg}$. Esta etapa es un eslabón fundamental en el sistema de producción animal, que permite el abastecimiento de terneros para recría y engorda.

Insertos en este complejo escenario surgen las exportaciones como una alternativa real y atractiva de solución a la crisis del sector. Esta alternativa permitirá a los pequeños productores crianceros reorientar su negocio a un atractivo circuito exportador.

La apertura económica del país y los acuerdos comerciales que se han firmado estos últimos años, sumado a las ventajas que le otorgan a Chile el estatus sanitario como uno de los países con mayor sanidad del mundo (es libre de fiebre aftosa y encefalopatía espongiforme bovina) han puesto al rubro de la carne bovina en una situación de amplia proyección, de mejores oportunidades y posibilidades de participación en mercados internacionales sofisticados y de altos precios. Así, Chile puede acceder a mercados exigentes y comercializar los productos pecuarios a mejores precios y condiciones que en el mercado interno (Klee, 2002).
De este modo y considerando la apertura de la economía nacional, las posibilidades de acceder al mercado externo se acrecientan con cierta solidez. Sin embargo, este mercado exige el cumplimiento de normas de calidad del producto, proceso que en este caso se inicia con la incorporación del predio en el registro de Planteles de Animales Bajo Certificación Oficial (PABCO) del Servicio Agrícola Ganadero (SAG) del Ministerio de Agricultura de Chile.

Con todas estas condiciones, comienzan a surgir numerosos inconvenientes para los pequeños productores crianceros, quienes necesariamente para exportar deberán incorporar sus predios ganaderos al programa $\mathrm{PABCO}$ y principalmente deberán mejorar las ineficiencias de sus producciones. Sin embargo, esta certificación abre oportunidades a la Agricultura Familiar Campesina (AFC), para adoptar las medidas necesarias e integrarse a la cadena de exportación a través de la venta de terneros certificados.

Es así como para aprovechar las oportunidades que ofrece el nuevo frente de producción impulsado por las posibilidades de exportar, obligará al sector a una adecuación a las nuevas exigencias y se deberán realizar ajustes estructurales que optimicen el funcionamiento de la cadena productiva (Catrileo, 2005). Se requiere un reordenamiento de la producción, especialmente en su fase primaria y se hace clave bajar los costos de los pequeños productores para que logren precios competitivos.

En este escenario, la presente investigación pretende elaborar un modelo de costos eficientes que permita a los pequeños productores crianceros ubicados desde la VII a la X regiones, "conocer el nivel de eficiencia que poseen sus costos", sobre la base de una comparación con los costos de sus pares, específicamente con aquellos productores que definen la frontera de mejor práctica. Todo esto con el objetivo de proporcionarles una ayuda técnica para mejorar sus producciones y darles mayor eficiencia tanto productiva como económica.

\section{MATERIALES Y MÉTODOS}

Para determinar los costos productivos asociados al sistema productivo de crianza, se aplicó una encuesta a través de entrevistas personales a 360 pequeños productores crianceros adscritos al Sistema de Planteles de Animales Bajo Certificación Oficial (PABCO) de las regiones más importantes en producción de ganado bovino en Chile, es decir, desde la VII a la $\mathrm{X}$ regiones (INE, 1997). 
Para determinar las variables relevantes que influyen en los costos de producción de los sistemas crianceros, se hizo un análisis detallado de las encuestas. De esta forma se seleccionaron las variables principales que son determinantes en este tipo de sistema productivo y que sirvieron de base para la siguiente etapa, es decir, se seleccionaron las "salidas" (output) y "entradas" (inputs) para realizar el análisis de eficiencia.

Para determinar y analizar las mejores prácticas productivas y determinar los costos eficientes en el sistema productivo de crianza se usó el principio de eficiencia comparativa o benchmarking. En líneas generales, la eficiencia comparativa consiste en la determinación de los costos eficientes (o relaciones de insumo productos eficientes) de una unidad productiva, sobre la base de una comparación con los costos de sus pares y, más específicamente, con aquellos productores que parecen definir la frontera de "mejor práctica".

Se identificaron los productores más eficientes en la producción de terneros al destete, y sobre la base de estos productores se identificaron los estándares técnicos y físicos para construir un productor modelo. Para tales efectos se realizó un análisis de la eficiencia global de los productores a partir del Análisis Envolvente de Datos (DEA). El análisis DEA se llevó a cabo con un software llamado EMS (Efficiency Measurement System), versión 1.3. Este software fue creado por Holger Scheel, de la Universidad de Dortmund, Alemania en el año 2000 y se puede obtener de la dirección electrónica http://www.wiso. uni-dortmund.de/lsfg/or/scheel/ems/.

Este software, partiendo de los insumos y productos, provee un ordenamiento de los agentes otorgándoles un puntaje de eficiencia relativa. De esta forma, los agentes que obtengan el mayor nivel de producto con la menor cantidad de insumos serán los más eficientes del grupo y, por ende, obtendrán los puntajes más altos.

Contraria a las metodologías paramétricas que se centran en el comportamiento de los productores haciendo referencia al promedio, la metodología DEA evalúa la eficiencia de un agente refiriéndose al "mejor" productor. Este modelo parte del comportamiento de una unidad tomadora de decisiones (DMU por sus siglas en inglés) y lo compara con el comportamiento de un productor "virtual" el cual es el resultado de la combinación de las mejores cualidades del resto de los DMU's considerados.

DEA es una metodología de puntos extremos, la cual parte de la posibilidad teórica de combinar las mejores características de dos o más productores para lograr un productor "virtual" que sea eficiente. Si se consideran dos productores con distintos niveles de insumos y de producción, el DEA asume que el primer agente puede cambiar su forma de producción y lograr los resultados del segundo y viceversa. El modelo realiza un proceso de optimización para cada agente en consideración.

Para ello construye un conjunto de referencia (peer o productor virtual). Si este productor virtual logra un rendimiento mayor con menos insumos, o si logra la misma producción con menos insumos, entonces el DMU considerado será ineficiente.

Una vez hecho esto, el modelo compara el DMU con ese conjunto de referencia y le otorga un puntaje. Un productor eficiente obtendrá un puntaje de $100 \%$.

En el Análisis de Eficiencia se evaluaron tres muestras: una muestra global (MG) conformada por 119 productores, una segunda muestra (MC) con 56 productores dedicados exclusivamente a la crianza de terneros y una tercera muestra (MCL) constituida por 63 productores de leche y subproducto la crianza de terneros.

Para cada muestra se aplicó un modelo orientado a los insumos (modelo input orientado) con dos variaciones, una con rendimientos constantes a escala (CCR), denominado así por haber sido desarrollado por Charnes, Cooper y Rhodes en 1978 y la otra con rendimientos variables a escala (BCC), denominado así por haber sido desarrollado por Banker, Charnes y Cooper en 1989. Los rendimientos a escala indican los incrementos de la producción que son resultado del incremento de todos los factores de producción en el mismo porcentaje y pueden ser: constantes (el incremento porcentual del output es igual al incremento porcentual de los recursos productivos), crecientes (el incremento porcentual del output es mayor que el incremento porcentual de los factores) o decrecientes (el incremento porcentual del output es menor que el incremento porcentual de los inputs).

En total se obtuvieron tres grupos de puntajes de eficiencia a partir de los cuales se determinaron los respectivos indicadores de eficiencia de escala.

El modelo de estimación de costos eficientes propuesto en este trabajo se fundamenta en la aplicación del concepto de eficiencia comparativa o benchmarking. Para tal efecto, se diseñó un sistema de crianza eficiente que incorpora las mejores prácticas productivas y las características técnicas de los productores crianceros eficientes detectados en la etapa anterior. 
En la lógica de este modelo, el costo total y medio de producción, medido en términos del peso vivo total de los terneros, se determina, en primer lugar, dimensionando los requerimientos físicos de los recursos (mano de obra, insumos agrícolas, superficie de praderas, cercos, corrales, etc.) que se requieren para producir una determinada cantidad de kilogramos de ternero, para luego valorizar los recursos e infraestructura mediante la aplicación de estándares de costos y precios de mercado de materiales, insumos y servicios.

En la construcción del modelo se identificaron y validaron los ítems de costos que deben financiarse en la explotación de estos sistemas de crianza. Para ello, se consideró como referencia la información contenida en la encuesta de este estudio, artículos e investigaciones de costos realizadas por organismos como el Instituto Nacional de Estadísticas y el Servicio Agrícola Ganadero del año 2004.

\section{RESULTADOS Y DISCUSIÓN}

Se realizó una revisión y análisis de los registros de las 360 encuestas, y se descartaron todos aquellos productores que no declararon información respecto de la cantidad de terneros producidos en el año, ya que el objetivo del sistema de crianza es la producción de terneros anualmente. De esta forma, la muestra se redujo a 119 productores.

Como se observa en el Cuadro 1, la muestra está conformada por 56 productores dedicados exclusivamente a la crianza de terneros y localizados principalmente en las regiones del Maule y Bío-Bío. Así también, la muestra contiene 63 productores dedicados a la producción conjunta de leche y crianza de terneros, los cuales se encuentran localizados principalmente en las regiones de La Araucanía, Los Lagos y Los Ríos.

En el Cuadro 1 se muestra la distribución de los productores por región y clasificados según tipo de sistema productivo: crianza y lechería-crianza.

Considerando el estadígrafo de la media, se analizaron las variables técnicas y económicas de los productores de la muestra según región. De acuerdo a estas variables los productores se caracterizan por:

a. Un inventario de vacas que en promedio asciende a 21 animales. Esta cifra está por sobre el promedio nacional que se estima para esta actividad, el cual es de 20 vacas por rebaño (Navarro, 2005).
CUADRO 1

DISTRIBUCIÓN DE PRODUCTORES POR REGIÓN Y TIPO DE SISTEMA PRODUCTIVO

\begin{tabular}{c|c|c|c}
\hline \multirow{2}{*}{ Región } & \multicolumn{3}{|c}{ Sistema Productivo } \\
\cline { 2 - 4 } & Crianza & Leche-Crianza & Total \\
\hline VII & 16 & 2 & 18 \\
\hline VIII & 24 & 2 & 26 \\
\hline IX & 0 & 38 & 38 \\
\hline X & 4 & 7 & 11 \\
\hline XIV & 12 & 14 & 26 \\
\hline Total & $\mathbf{5 6}$ & $\mathbf{6 3}$ & $\mathbf{1 1 9}$ \\
\hline
\end{tabular}

b. El tamaño de las praderas destinadas al pastoreo de los animales es en promedio de 25 hectáreas, salvo la superficie media de las praderas de la $\mathrm{X}$ Región que duplican en tamaño la superficie media de los productores de las otras regiones.

c. Una intensidad en el uso de las praderas, representada por la carga animal (Número de animales ha-1) del orden de 2,05 animales por hectárea. Este indicador está por debajo de la media, 2,05 animales por hectárea, en las regiones VIII y X, las cuales ascienden a 1,87 y 1,93 respectivamente.

d. Un número de terneros nacidos y vivos que en promedio asciende a 16 animales. Esto indica que el porcentaje de parición de las vacas es de aproximadamente un $76 \%$, es decir, inferior al porcentaje promedio de parición en nuestro país, el cual es de un $85 \%$ (Navarro, 2005); el porcentaje de mortalidad es del $24 \%$ durante la gestación, el parto o en las dos primeras semanas de vida. Se puede apreciar además que en cuanto a la eficiencia reproductiva los productores más eficientes se encuentran en la Región de Los Lagos.

e. Un promedio total de kilogramos de ternero producidos (peso vivo) al año de $3.585 \mathrm{~kg}$. Los productores más eficientes en cuanto a kilogramos de ternero producidos al año se encuentran en la $\mathrm{X}$, VIII y IX regiones.

f. Un precio de venta promedio por kilogramo de ternero de $\$ 615$. Este es el precio promedio de venta a febrero del 2007. Su equivalente en dólares a esta fecha es de US\$1,13. Hay que considerar que este precio depende fundamentalmente del lugar de venta de los terneros, sin embargo, los productores de la muestra venden sus terneros principalmente en ferias de su sector, por lo que los precios obtenidos son bastante homogéneos para todos. Los productores que venden a un 
precio mayor a la media son los de la X, VII y IX regiones.

g. Un costo directo unitario promedio por $\mathrm{kg}$ de $\$ 494$. Este costo unitario es bastante alto si se tiene en cuenta que los productores de la IX Región tienen un costo de $\$ 289 \mathrm{~kg}-1$, lo que demuestra el alto grado de heterogeneidad presente en estos sistemas productivos. Se puede indicar además que con estos costos directos las ganancias de los productores son muy bajas si se tiene en cuenta que en promedio el precio de venta es de $\$ 615$ $\mathrm{kg}-1$. Para disminuir los costos directos, los productores deben mejorar en aspectos como compra de alimentos (fardos, ensilaje, concentrados, sales minerales, entre otros), manejo sanitario (compra de vitaminas, vacunas y antiparasitarios), manejo reproductivo (compra de dosis para la inseminación artificial) y compra de fertilizantes para sus praderas. Los productores más eficientes en cuanto al costo unitario son los de la IX región, los cuales tienen claramente un costo mucho menor al de sus pares en las otras regiones.

Las características de los productores según tipo de sistema productivo, sobre la base de variables técnico-económicas, son las siguientes:

a. Un inventario de vacas que en promedio asciende a 22 animales en el caso del sistema de crianza y 19 animales para el sistema de lechería y crianza. Esto comparado con el promedio nacional de 20 vacas por rebaño, muestra que para ambos sistemas el número de vacas presentes es primordial para el desarrollo de la actividad y no es una variable que diferencie ambos sistemas productivos.

b. El tamaño de las praderas destinadas al pastoreo de los animales es en promedio de 27 hectáreas para el sistema de crianza y 24 hectáreas para el sistema de lechería y crianza. En cuanto a las praderas el tamaño es bastante homogéneo en ambos sistemas productivos. Sin embargo se puede decir que más que el número de hectáreas de praderas, importa si éstas son mayormente naturales o artificiales, ya que de esto depende el porcentaje de materia seca que se puede obtener de ellas. Se entiende por materia seca todos aquellos componentes de la pradera como hidratos de carbono, lípidos, proteínas, sales minerales y vitaminas que son fundamentales en la alimentación de los animales.

c. Una intensidad en el uso de las praderas, representada por la carga animal de 2,0 para el sistema de crianza y 2,1 para el sistema de lechería y crianza.
Es decir, ambos sistemas permiten un pastoreo que maximiza el consumo de materia seca de la pradera, sin dañar la capacidad de recuperación después de ser utilizada, así como la persistencia de la misma.

d. Un número de terneros nacidos vivos que en promedio asciende a 16 animales en ambos sistemas. El porcentaje de parición de las vacas y el porcentaje de mortalidad es igual en ambos sistemas ( $76 \%$ y $24 \%$ respectivamente).

e. Un promedio total de kilogramos de ternero producidos (peso vivo) al año de $3.807 \mathrm{~kg}$ en el sistema de crianza y $3.388 \mathrm{~kg}$ en el sistema de lechería y crianza. Lo anteriormente señalado muestra que los productores dedicados sólo a la crianza de terneros pueden lograr un mayor número de kilogramos producidos debido principalmente a que las vacas no son ordeñadas y los terneros se alimentan principalmente de leche, lo cual provoca que estos tengan una ganancia de peso mayor a aquellos terneros de los sistemas productivos de lechería y crianza, que deben alimentarse principalmente de alimentos suplementarios.

f. Un precio de venta promedio por $\mathrm{kg}$ de ternero producido de $\$ 724$ en el sistema de crianza y $\$ 445$ en el sistema de lechería y crianza. Aquí vemos claramente que los productores dedicados sólo a la crianza de terneros obtienen un precio mucho mayor y por ende mejores ingresos. Esto puede deberse fundamentalmente al peso de venta de los terneros, que como se mencionó anteriormente parece ser mayor en el sistema de crianza.

g. Un costo directo unitario promedio por $\mathrm{kg}$ de $\$ 650$ en el sistema de crianza y $\$ 355$ en el sistema de lechería y crianza. Se ve claramente que los productores más eficientes y con menores costos unitarios son los productores que se dedican al sistema multipropósito (lechería y crianza). Esto se debe principalmente a los costos compartidos entre los productos, es decir, no hay una asignación directa de los costos incurridos para cada actividad, sino que se consideran como un todo.

\section{Selección de Variables para el Análisis de Eficiencia}

Se definió como única "salida" (output) el peso total de los terneros producidos en un año en el predio. Se desestimó considerar el ingreso por venta de ternero para evitar que las mediciones de eficiencia se vieran influenciadas por factores exógenos no controlables por los productores. Por tal razón, se consideró como único producto una medida de la 
cantidad física, el peso total de los terneros producidos (kilogramos/año).

De las potenciales variables de "entrada" (inputs), se seleccionaron: el número de vacas, la superficie de las praderas destinadas al pastoreo de los animales y los costos directos totales incurridos en la explotación de los predios. Los fundamentos de esta elección son los siguientes:

a. En general, las vacas tienen una incidencia directa tanto en el nivel de producción de leche como en la cantidad de terneros que potencialmente pueden ser vendidos en un año. Así también, este factor junto con la tierra son los recursos de capital más importantes en este tipo de explotaciones ganaderas.

b. De igual forma y por el bajo nivel de tecnificación presente en estos sistemas, se espera que la cantidad y calidad de las praderas destinada al pastoreo sea un factor determinante en la ganancia de peso de los animales.

c. Se seleccionaron los costos directos como una forma de representar el costo de los recursos asociados a diversas actividades realizadas en el predio, tales como el manejo reproductivo y sanitario de los animales, la fertilización de praderas, el valor de concentrados y otros alimentos, entre otras.

Cabe señalar que se desestimó incluir variables representativas del activo fijo porque se estima que su cuantía es irrelevante, debido el bajo grado de tecnificación presente en este tipo de actividad productiva (Klee, 2004).

Las expresiones matemáticas utilizadas para cuantificar estas variables se explican a continuación:

a) Kilogramos de ternero producido (KGPT): corresponde a los kilogramos de terneros al destete, y se obtiene mediante la siguiente expresión:

$$
K G P T=(N P-N T M) \cdot P M D \quad \text { Ecuación } 1.1
$$

donde: $\mathrm{NP}=$ Número promedio de partos en el plantel al año; NTM = Número de terneros que mueren después del segundo día de vida; $\mathrm{PMD}=$ Peso promedio al destete expresado en kilogramos.

b) Número de vacas (NVAC): Corresponde al número total de vacas presentes en el plantel al inicio del año t. Se obtiene a partir de la siguiente expresión:

$$
N V A C=N V_{t}+N V V_{t}-N V C_{t} \quad \text { Ecuación } 1.2
$$

donde: $N V A C=$ Número de vacas al final del año t-1 $o$ al inicio del año t;

$N V_{t} \quad=$ Número de vacas al final del año t;

$N V V_{t}=$ Número de vacas vendidas en el año t;

$N V C_{t}=$ Número de vacas compradas en el año $\mathrm{t}$.

c) Superficie de praderas (ha): expresada en hectáreas y se obtiene de la suma de la superficie de las praderas sembradas, mejoradas y naturales.

d) Costos directos totales del predio (CD). Corresponde al gasto anual del predio, el cual se obtiene sumando los costos de mano de obra, asistencia técnica, alimentación (costo de los fardos, ensilaje, concentrados, otros), fertilización de praderas, manejo sanitario y manejo reproductivo, entre otros.

Como se ve en el Cuadro 2, existe una gran dispersión de tamaños en la muestra, tanto en lo referido a los kilos de terneros producidos al año, como al número de vacas y también a la superficie $\mathrm{y}$ costos directos.

\section{Análisis de Eficiencia}

Los resultados obtenidos en la muestra total (MG), reflejados en el Cuadro 3, indican que existen sólo 7 productores eficientes que operan con rendimientos constantes de escala, lo que representa un $6 \%$ de los productores totales de la muestra. Por el contrario, el número de productores eficientes bajo rendimientos variables a escala aumentó considerablemente.

CUADRO 2

ESTADÍSTICAS DE LAS VARIABLES SELECCIONADAS. MES, AÑO

\begin{tabular}{l|c|c|c|c}
\hline \multirow{2}{*}{ Estadigrafos } & Output & \multicolumn{3}{|c}{ Input } \\
\cline { 2 - 5 } & $\begin{array}{c}\text { Terneros } \\
(\mathrm{kg} \text { año-1) }\end{array}$ & $\begin{array}{c}\text { Vacas } \\
\left(N^{\circ}\right)\end{array}$ & $\begin{array}{c}\text { Superficie } \\
\text { (ha praderas) }\end{array}$ & $\begin{array}{c}\text { Costos Directos } \\
(\$)\end{array}$ \\
\hline Media & 3.585 & 21 & 25 & 1.585 .588 \\
\hline Desviación Estándar & 2.629 & 12 & 21 & 1.498 .874 \\
\hline Valor Mínimo & 300 & 4 & 4 & 68.012 \\
\hline Valor Máximo & 16.380 & 59 & 140 & 6.972 .012 \\
\hline
\end{tabular}


CUADRO 3

ESTADÍSTICAS DE LOS PUNTAJES

DE EFICIENCIA - MUESTRA TOTAL (MG)

\begin{tabular}{l|c|c|c}
\hline \multirow{2}{*}{ Estadisticas } & \multicolumn{3}{|c}{ Orientación Insumos } \\
\cline { 2 - 4 } & $\theta(C C R)$ & $\theta(B C C)$ & $\theta(E E)$ \\
\hline Media & $53 \%$ & $66 \%$ & $81 \%$ \\
\hline Desviación estándar & $22 \%$ & $22 \%$ & $17 \%$ \\
\hline Valor Mínimo & $6 \%$ & $12 \%$ & $15 \%$ \\
\hline $\mathrm{N}^{\circ}$ Productores eficientes & 7 & 17 & 8 \\
\hline$\%$ Productores eficientes & $6 \%$ & $14 \%$ & $7 \%$ \\
\hline
\end{tabular}

El promedio de los puntajes de eficiencia fue $53 \%$ para el modelo con rendimientos constantes de escala (CRR) y $66 \%$ para el modelo con rendimientos variables de escala (BCC). Lo anterior demuestra el alto nivel de ineficiencia de los productores de la muestra. Existe, por tanto, una posibilidad de ahorro de recursos, sin modificar el nivel de producción de $\mathrm{kg}$ de terneros producido, de un $47 \%$. En otras palabras, existe un grado de subutilización de los recursos que en promedio asciende a ese porcentaje.

$\mathrm{Al}$ descomponer la eficiencia global $\theta$ (CCR) en sus dos componentes, eficiencia técnica (BCC) y eficiencia de escala (EE), se observa que los productores presentan un mayor nivel de ineficiencia técnica (34\%) con respecto a la ineficiencia debido a la escala (19\%). Es decir, los productores están siendo más ineficientes en aspectos relacionados al manejo de la producción, que a aspectos de tamaño en sus explotaciones.

En general, se obtuvo que el $78 \%$ de los productores operan con rendimientos constantes de escala, $16 \%$ con rendimientos crecientes de escala y $6 \%$ con rendimientos decrecientes de escala. Es decir, para la mayoría de los productores el rendimiento no depende de la escala de operación. En el Cuadro 3 se muestran los puntajes obtenidos.

Cuando se agrupan los resultados de la muestra total (MG) por región de origen, mostrados en el Cuadro 4, se puede apreciar que los productores más eficientes se encuentran ubicados en las regiones del Maule (VII), La Araucanía (IX) y del Bío-Bío (VIII), con un nivel de eficiencia global promedio (CCR) cercana al 58\%. En el Cuadro 5 se puede ver además que el origen de las ineficiencias es atribuible, en gran parte, a un manejo productivo deficiente $\mathrm{y}$, en menor medida, por razones de tamaño o escala. Si los productores corrigieran sus deficiencias técnicas, podrían lograr un ahorro de recursos cercano al 34\%.
Cabe destacar que la Región de Los Ríos alcanzó el puntaje de eficiencia global más bajo (40\%), obteniendo el segundo puntaje más bajo en eficiencia técnica y el primer puntaje más bajo en eficiencia de escala. En cambio, los productores de la VIII Región obtuvieron el puntaje de eficiencia de escala más alto. Esto indicaría que la gran mayoría de los productores de esta región están operando a una escala óptima de producción, sin embargo, con una alta ineficiencia técnica (41\%).

En el Cuadro 4 se presenta la distribución de puntajes obtenidos por región.

Analizando los resultados en función del tipo de sistema productivo, como se ve en el Cuadro 5, se tiene que sólo 7 productores son eficientes bajo el modelo de rendimientos constantes de escala y 17 productores eficientes bajo el modelo con rendimientos variables de escala. En ambos casos, el $86 \%$ y $59 \%$ de los productores eficientes están asociados a un sistema de producción del tipo mixto (leche y carne).

Si se compara a los productores dedicados al sistema de Lechería y Crianza con aquellos dedicados sólo a la Crianza, se ve que en promedio los puntajes de eficiencia fueron $52 \%$ y $55 \%$ respectivamente, lo que demuestra el alto nivel de ineficiencia presente en ambos sistemas. En el caso del sistema de lechería y crianza se encuentran 6 productores con una eficiencia del $100 \%$. Por el contrario, en el caso del sistema de crianza se encuentra sólo un productor con una eficiencia del $100 \%$. Los resultados obtenidos indican que el sistema mixto leche-crianza presenta el mayor número de productores eficientes. Esto puede estar explicado por las economías de ámbito existente en los sistemas mixtos de producción, ya que los costos se financian entre ambas actividades.

Se observa también que los productores con mayor nivel de ineficiencia técnica (36\%) son aquellos dedicados al sistema de crianza. Por el contrario los

CUADRO 4

PUNTAJES DE EFICIENCIA PROMEDIO POR REGIÓN

\begin{tabular}{c|c|c|c}
\hline & \multicolumn{3}{|c}{ Orientación Insumos } \\
\hline Región & $\theta(C C R)$ & $\theta(B C C)$ & $\theta(E E)$ \\
\hline VII & $61 \%$ & $76 \%$ & $82 \%$ \\
\hline VIII & $55 \%$ & $59 \%$ & $91 \%$ \\
\hline IX & $59 \%$ & $74 \%$ & $80 \%$ \\
\hline X & $48 \%$ & $56 \%$ & $87 \%$ \\
\hline XIV & $40 \%$ & $57 \%$ & $71 \%$ \\
\hline Promedio & $53 \%$ & $66 \%$ & $81 \%$ \\
\hline
\end{tabular}


CUADRO 5

RESUMEN DE PUNTAJES SEGÚN SISTEMA PRODUCTIVO

\begin{tabular}{l|c|c|c|c|c|c}
\hline & \multicolumn{3}{|c|}{ Crianza } & \multicolumn{3}{c}{ Lecheria-Crianza } \\
\cline { 2 - 7 } & $\theta(C C R)$ & $\theta(B C C)$ & $\theta(E E)$ & $\theta(C C R)$ & $\theta(B C C)$ & $\theta(E E)$ \\
\hline Media & $55 \%$ & $64 \%$ & $87 \%$ & $52 \%$ & $67 \%$ & $76 \%$ \\
\hline Desviación estándar & $20 \%$ & $22 \%$ & $13 \%$ & $23 \%$ & $22 \%$ & $19 \%$ \\
\hline Valor Mínimo & $14 \%$ & $20 \%$ & $58 \%$ & $6 \%$ & $12 \%$ & $15 \%$ \\
\hline $\mathrm{N}^{\text {o Productores eficientes }}$ & 1 & 7 & 1 & 6 & 10 & 6 \\
\hline$\%$ Productores eficientes & $14 \%$ & $41 \%$ & $13 \%$ & $86 \%$ & $59 \%$ & $75 \%$ \\
\hline
\end{tabular}

CUADRO 6

RESUMEN DE MEJORAS POTENCIALES POR REGIÓN

\begin{tabular}{|c|c|c|c|c|c|c|c|}
\hline \multirow{2}{*}{\multicolumn{2}{|c|}{ Variable }} & \multicolumn{5}{|c|}{ Región } & \multirow{3}{*}{$\begin{array}{c}\begin{array}{c}\text { Total } \\
\text { general }\end{array} \\
2.440\end{array}$} \\
\hline & & \multirow{2}{*}{$\begin{array}{l}V I I \\
348\end{array}$} & \multirow{2}{*}{$\begin{array}{l}\text { VIII } \\
563\end{array}$} & \multirow{2}{*}{$\frac{I X}{799}$} & \multirow{2}{*}{$\begin{array}{c}X \\
262\end{array}$} & \multirow{2}{*}{$\frac{X I V}{468}$} & \\
\hline \multirow{3}{*}{ Número Vacas } & Actual & & & & & & \\
\hline & Objetivo & 316 & 281 & 440 & 130 & 253 & 1.419 \\
\hline & Mejora potencial (\%) & $-9,2 \%$ & $-50,1 \%$ & $-44,9 \%$ & $-50,5 \%$ & $-46,0 \%$ & $-41,8 \%$ \\
\hline \multirow{3}{*}{ Superficie Praderas (ha) } & Actual & 338 & 738 & 941 & 472 & 524 & 3.011 \\
\hline & Objetivo & 317 & 345 & 557 & 162 & 257 & 1.639 \\
\hline & Mejora potencial (\%) & $-6,1 \%$ & $-53,2 \%$ & $-40,8 \%$ & $-65,7 \%$ & $-50,9 \%$ & $-45,6 \%$ \\
\hline \multirow{3}{*}{ Costos Directos (\$) } & Actual & 12.467 .207 & 62.466 .859 & 50.694 .498 & 30.580 .670 & 32.475 .693 & 188.684 .927 \\
\hline & Objetivo & 12.030 .149 & 17.417 .464 & 22.075 .790 & 7.466 .156 & 14.333 .707 & 73.323 .265 \\
\hline & Mejora potencial (\%) & $-3,5 \%$ & $-72,1 \%$ & $-56,5 \%$ & $-75,6 \%$ & $-55,9 \%$ & $-61,1 \%$ \\
\hline
\end{tabular}

productores con mayor nivel de ineficiencia debido a la escala (24\%), son aquellos dedicados al sistema multipropósito.

En el Cuadro 5 se presenta la comparación de los puntajes promedios obtenidos por sistema productivo.

Una de las características importantes de este tipo de análisis es que permite descomponer la ineficiencia de acuerdo a los insumos utilizados, lo que permitiría encontrar mejoras potenciales. $\mathrm{La}$ mejora (reducción de insumos y/o incremento de producto) que debería experimentar cada productor ineficiente para convertirse en eficiente puede calcularse al comparar los valores observados y objetivos de éstos.

En este sentido, en el Cuadro 6 se puede observar que en promedio el recurso "superficie de praderas" está siendo subutilizado en un $45,6 \%$, lo que es una cifra relativamente alta por la importancia que tiene este factor en la producción tanto de leche como de carne. La Región del Maule es la región que presenta el menor grado de subutilización de este recurso $(6,1 \%)$, es decir, es la región más eficiente en este aspecto y con el número de hectáreas actuales podrían aumentar su producción en ese porcentaje.

Del mismo modo, se observa un ahorro potencial o un grado de subutilización del 41,8\% en el número de vacas que poseen los productores, siendo nuevamente la VII Región la que presenta un mejor grado de utilización del recurso "vacas".

En lo que respecta a los costos directos de explotación, se encontró que existe una potencial reducción cercana al $61,1 \%$. Se mantiene en este caso la tendencia de la VII Región, ya es aquella que presenta los menores ajustes. En este caso vemos que la mejora potencial de esta región es muy inferior al resto de las regiones.

Se puede apreciar que la región más eficiente en todos los aspectos considerados es la Región del Maule, la cual es seguida lejanamente por la Región de La Araucanía.

En el Cuadro 6 se presentan las potenciales mejoras resultantes de aplicar el modelo.

$\mathrm{Al}$ organizar las mejoras potenciales por tipo de sistema productivo, como se ve en el Cuadro 7, se obtuvo lo siguiente: 
CUADRO 7

RESUMEN DE MEJORAS POTENCIALES SEGÚN SISTEMA PRODUCTIVO

\begin{tabular}{|c|c|c|c|c|}
\hline \multirow{2}{*}{\multicolumn{2}{|c|}{ Variable }} & \multicolumn{3}{|c|}{ Sistema Productivo } \\
\hline & & \multirow{2}{*}{$\begin{array}{c}\text { Crianza } \\
1.233\end{array}$} & \multirow{2}{*}{$\begin{array}{c}\text { Lecheria-Crianza } \\
1.207\end{array}$} & \multirow{2}{*}{$\begin{array}{c}\text { Total General } \\
2.440 \\
\end{array}$} \\
\hline \multirow{3}{*}{ Número Vacas } & Actual & & & \\
\hline & Objetivo & 721 & 699 & 1.419 \\
\hline & Mejora potencial (\%) & $-41,56 \%$ & $-42,10 \%$ & $-41,83 \%$ \\
\hline \multirow{3}{*}{ Superficie Praderas (ha) } & Actual & 1.497 & 1.514 & 3.011 \\
\hline & Objetivo & 798 & 841 & 1.639 \\
\hline & Mejora potencial (\%) & $-46,69 \%$ & $-44,50 \%$ & $-45,59 \%$ \\
\hline \multirow{3}{*}{ Costos Directos (\$) } & Actual & 84.982 .898 & 103.702 .029 & 188.684 .927 \\
\hline & Objetivo & 35.676 .917 & 37.646 .349 & 73.323 .265 \\
\hline & Mejora potencial (\%) & $-58,02 \%$ & $-63,70 \%$ & $-61,14 \%$ \\
\hline
\end{tabular}

CUADRO 8

VARIABLES TÉCNICAS Y ECONÓMICAS EN FUNCIÓN DE LA EFICIENCIA GLOBAL

\begin{tabular}{l|c|c|c|c}
\hline \multirow{2}{*}{ Casos } & \multicolumn{4}{|c}{ Puntajes de eficiencia (CCR) } \\
\cline { 2 - 5 } & $<40$ & $40-59$ & $60-89$ & $90-100$ \\
\cline { 2 - 5 } & 29 & 53 & 22 & 15 \\
\hline
\end{tabular}

Variables Técnicas

\begin{tabular}{l|c|c|c|c}
\hline $\mathrm{N}^{\circ}$ Vacas & 22 & 18 & 23 & 23 \\
\hline $\mathrm{Kg}$ de Ternero Producidos & 2.150 & 3.270 & 4.765 & 5.743 \\
\hline $\mathrm{N}^{\mathrm{o}}$ Terneros & 13 & 14 & 20 & 23 \\
\hline Peso Terneros & 172 & 224 & 235 & 255 \\
\hline $\mathrm{N}^{\circ}$ Animales & 42 & 37 & 47 & 52 \\
\hline Ha Praderas & 28 & 27 & 22 & 20 \\
\hline Indicadores de productividad & 1,86 & 1,76 & 2,3 & 3,1 \\
\hline Carga $(\mathrm{Cab} / \mathrm{ha})$ & 0,61 & 0,83 & 1,0 & 1,1 \\
\hline $\mathrm{N}^{\circ}$ Terneros/Vaca &
\end{tabular}

Variables Económicas

\begin{tabular}{l|l|l|l|l}
\hline Precio Ternero $(\$ / \mathrm{kg})$ & 581 & 603 & 610 & 729 \\
\hline Costo Unitario Total $(\$ / \mathrm{kg})$ & 580 & 561 & 377 & 266 \\
\hline
\end{tabular}

a. Los productores dedicados al sistema productivo de crianza presentan un mejor nivel de utilización del recurso "vacas" en comparación con sus pares que están dedicados al sistema multipropósito. En el caso del recurso "superficie de praderas" son los productores dedicados al sistema de lechería y crianza los que presentan un mejor nivel de utilización de este recurso.

b. Ambos sistemas de producción presentan costos directos de explotación excesivamente altos, sin embargo los que hacen un mejor uso de este recurso, es decir son más eficientes en este aspecto, son los productores dedicados al sistema de crianza los cuales podrían ajustar este recurso en un 58,02\%.
En el Cuadro 8 se analiza la relación entre la eficiencia y la variación de las principales variables técnicas y económicas. Se agruparon los productores en cuatro categorías de puntajes de eficiencia técnica global (CCR), siendo los intervalos: $<40$, de 40 a 52 , de 53 a 65 y de 66 a 100, donde los productores ubicados en el intervalo $<40$, son aquellos productores menos eficientes y los ubicados en el intervalo 66 a 100 son aquellos productores más eficientes. De esto se obtuvo lo siguiente:

a. El grupo de menor eficiencia $(<40)$ coincide con ser el grupo de menor escala medida en términos de las variables técnicas como los kilos de terneros producidos, el número de terneros y peso de los 
terneros. De igual forma, este grupo presenta la más baja productividad medida por el índice de número de terneros por vaca. En cuanto a las variables económicas, se tiene que en relación a los costos unitarios de explotación por kilo de ternero producido (PV), estos productores presentan el mayor costo total de producción de terneros, el cual asciende a $580 \$ /$ kilo.

b. Si se compara el grupo de menor eficiencia con el de mayor eficiencia, se observa que a medida que aumenta la eficiencia, el tamaño de las praderas disminuye y el número de animales aumenta. Lo que se ve reflejado en un aumento del $45 \%$ de la productividad de las praderas, alcanzando una carga animal de 2,7 (cab/ha).

c. Otro aspecto a destacar es que a medida que aumenta la eficiencia crece la escala de producción medida en términos de los kilos de terneros al destete, y el costo unitario se comporta de acuerdo a lo esperado, de manera inversa al aumento de escala, alcanzando un valor de 359 \$/kilos para los productores en el rango de mayor eficiencia. Es decir, se comporta claramente de acuerdo a la ley de economías de escala (a medida que se aumenta la cantidad producida, disminuyen los costos de producción).

En los Cuadros 9 y 10 se muestra la relación entre la eficiencia global y la variación de las principales variables técnicas y económicas por sistema productivo. De acuerdo a esto, se obtuvo lo siguiente: a. En el caso del sistema de lechería y crianza, los productores menos eficientes tienen un costo unitario total de $\$ 352 /$ kilo. En el caso de los productores con mayor eficiencia, éstos poseen un costo de $\$ 350 /$ kilo. Se ve claramente que entre ambos extremos no hay una gran diferencia en sus costos. Esto demuestra que en los sistemas de lechería y crianza los costos totales están distribuidos en ambas actividades, por lo que el costo unitario es mucho menor.

b. Por el contrario, en los sistemas de crianza, los productores menos eficientes tienen un costo unitario de $\$ 901 /$ kilo y los más eficientes un costo unitario de \$366/kilo. Se ve notoriamente que la diferencia entre ambos extremos es más del triple. Para los productores que sólo se dedican al sistema de crianza la situación es más compleja, ya que sus costos totales se distribuyen sólo en la actividad de vender sus terneros y se ve muy claramente cómo operan las economías de escala, es decir, en este tipo de sistema productivo influye fuertemente el tamaño de la explotación ganadera.

\section{CONCLUSIONES}

Este análisis, demostró el alto nivel de ineficiencia que presentan los pequeños productores de la muestra, independiente del sistema productivo al que se

CUADRO 9

VARIABLES TÉCNICAS Y ECONÓMICAS EN FUNCIÓN DE LA EFICIENCIA GLOBAL-SISTEMA LECHERÍA Y CRIANZA

\begin{tabular}{|c|c|c|c|c|}
\hline \multirow[b]{3}{*}{ Casos } & \multicolumn{4}{|c|}{ Puntajes de eficiencia (CCR) } \\
\hline & $<40$ & $40-52$ & $53-65$ & $66-100$ \\
\hline & 17 & 14 & 18 & 14 \\
\hline \multicolumn{5}{|l|}{ Variables Técnicas } \\
\hline $\mathrm{N}^{\mathrm{o}}$ Vacas & 20 & 12 & 23 & 21 \\
\hline Kg de Ternero Producidos & 1.889 & 2.092 & 4.118 & 5.564 \\
\hline $\mathrm{N}^{\mathrm{o}}$ Terneros & 13 & 10 & 17 & 24 \\
\hline Peso Terneros & 150 & 213 & 229 & 241 \\
\hline $\mathrm{N}^{\mathrm{o}}$ Animales & 40 & 28 & 44 & 52 \\
\hline Ha Praderas & 27 & 24 & 21 & 24 \\
\hline \multicolumn{5}{|l|}{ Indicadores de productividad } \\
\hline Carga (Cab/ha) & 1,91 & 1,68 & 2,13 & 2,54 \\
\hline $\mathrm{N}^{\circ}$ Terneros/Vaca & 0,65 & 0,87 & 0,82 & 1,22 \\
\hline \multicolumn{5}{|l|}{ Variables Económicas } \\
\hline Precio Ternero $(\$ / \mathrm{kg})$ & 610 & 679 & 588 & 701 \\
\hline Costo unitario total $(\$ / \mathrm{kg})$ & 352 & 524 & 231 & 350 \\
\hline
\end{tabular}


CUADRO 10

VARIABLES TÉCNICAS Y ECONÓMICAS EN FUNCIÓN DE LA EFICIENCIA GLOBAL-SISTEMA CRIANZA

\begin{tabular}{|c|c|c|c|c|}
\hline \multirow[b]{3}{*}{ Casos } & \multicolumn{4}{|c|}{ Puntajes de eficiencia (CCR) } \\
\hline & $<40$ & $40-52$ & $53-65$ & $66-100$ \\
\hline & 12 & 16 & 11 & 17 \\
\hline \multicolumn{5}{|l|}{ Variables Técnicas } \\
\hline $\mathrm{N}^{\mathrm{o}}$ Vacas & 26 & 18 & 23 & 22 \\
\hline Kg de Ternero Producidos & 2.519 & 3.408 & 4.005 & 4.963 \\
\hline $\mathrm{N}^{\mathrm{o}}$ Terneros & 12 & 15 & 18 & 20 \\
\hline Peso Terneros & 203 & 229 & 226 & 250 \\
\hline $\mathrm{N}^{\mathrm{o}}$ Animales & 45 & 37 & 45 & 47 \\
\hline Ha Praderas & 29 & 37 & 22 & 18 \\
\hline \multicolumn{5}{|l|}{ Indicadores de productividad } \\
\hline Carga (Cab/ha) & 1,79 & 1,27 & 2,24 & 2,82 \\
\hline $\mathrm{N}^{\circ}$ Terneros/Vaca & 0,54 & 0,82 & 0,82 & 0,94 \\
\hline \multicolumn{5}{|l|}{ Variables Económicas } \\
\hline Precio Ternero $(\$ / \mathrm{kg})$ & 540 & 588 & 564 & 636 \\
\hline Costo unitario total $(\$ / \mathrm{kg})$ & 901 & 908 & 441 & 366 \\
\hline
\end{tabular}

dediquen, sólo crianza o mixto (lechería-crianza). Además, demuestra que la mayor cantidad de productores eficientes están asociados a un sistema de producción del tipo mixto.

El sistema productivo es muy importante a la hora de calcular los costos unitarios totales, ya que aquellos productores dedicados al sistema de lechería y crianza poseen economías de ámbito, lo que permite que sus costos totales estén financiados por ambas actividades.

Por otro lado, los productores dedicados sólo a la crianza de terneros, a pesar de tener costos unitarios totales más altos, logran un mayor número de kilos producidos.

La ineficiencia detectada en ambos sistemas se debe principalmente a aspectos técnicos más que de escala, ya que los productores son ineficientes principalmente en el manejo de la producción (no poseen registros, tienen una baja capacidad de gestión, un bajo conocimiento tecnológico y un casi nulo conocimiento de los actuales requerimientos del mercado), más que en aspectos relacionados con el tamaño de sus explotaciones.

La variable más influyente en esta ineficiencia técnica es el costo directo de producción. Para disminuir estos costos directos, los productores deben mejorar en aspectos como la compra de alimentos, manejo sanitario, manejo reproductivo y compra de fertilizantes para sus praderas.
Los productores más eficientes de la muestra pertenecen a la Región del Maule (VII Región) y aquéllos más ineficientes se encuentran en la Región de Los Ríos (XIV Región), aspecto que llama la atención, debido a las conocidas aptitudes que presenta esta región para el desarrollo de la ganadería y el protagonismo económico que significa esta actividad para la zona.

Esta evaluación, además, permitió demostrar que al igual que en muchas otras áreas de la economía nacional, la ley de las economías de escala opera con éxito, ya que al aumentar las unidades producidas, tanto en el sistema de crianza como en el de lechería y crianza, los costos unitarios de producción disminuyen.

Para que este primer eslabón de la cadena productiva bovina mejore sus niveles de eficiencia, es recomendable proporcionar una asesoría constante por parte de técnicos y profesionales entendidos en el tema, aspecto donde las instituciones más importantes para el desarrollo y fomento de esta actividad deben cumplir un rol fundamental.

\section{RECONOCIMIENTOS}

Los autores agradecen el financiamiento aportado por el proyecto "Desarrollo de Modelos de Evaluación de Costos para Explotaciones Ganaderas de la Agri- 
cultura Familiar Campesina", de INDAP, que hizo posible la realización de esta investigación.

\section{REFERENCIAS}

CAtrileo, A. 2004. Buenas Prácticas Ganaderas. Nuevo Enfoque en la Producción de Carne. Rojas, C, editor. Manual de Producción de Bovinos de Carne para la VIII, IX y X Regiones. I ed., Temuco, INIA, pp. 11-30.

CATrileo, Adrián S. 2005. Producción y Manejo de Carne Bovina en Chile. Colección libros INIA N ${ }^{\circ} 16$. Instituto de Investigaciones Agropecuarias. Chile.

Coll, Vicente S.; Blasco, Ma Olga. 2006. Evaluación de la Eficiencia mediante el Análisis Envolvente de Datos. Universidad de Valencia. España.

FERRADA, S. 2004. Registros y Análisis Económico en Producción de Carne. Rojas, C, editor. Manual de Producción de Bovinos de Carne para la VIII, IX y X Regiones. I ed, Temuco, INIA, pp. 235-254.

GoIC, M; RoJAs, C. 2004. Sistemas de Crianza, Recría y Engorda en la Zona Sur. Rojas, C, editor. Manual de Producción de Bovinos de Carne para la VIII, IX y X Regiones. I ed, Temuco, INIA, pp. 107-120.

KLeE, Germán G. 2002. Producción Práctica de Carne Bovina, Sistema Vaca-Ternero. Boletín INIA N ${ }^{\circ} 93.206$ pp. Instituto de Investigaciones Agropecuarias. Chile.

KLeE, Germán G. 2004. Sistemas Vaca-Ternero y Recría-Engorda de Novillos. Boletín INIA No 117.128 pp. Instituto de Investigaciones Agropecuarias. Chile.
LEPORATI, M. 2005. El Plan Ganadero para la Agricultura Campesina: Incorporación de la Agricultura Campesina a los Procesos de Exportación, I ed., Santiago, ProChile, pp. 61-88.

Leporati, M; SÁez, L; Villalobos, P; Manríquez, R. 2005. Determinación de Costos de Producción en Planteles Bovinos de la Agricultura Familiar Campesina. X Congreso de Economistas Agrarios. Noviembre. Temuco. Chile.

Maino, Mario M.; Pittet, Julio D.; Bruna, Gastón D.; Henríquez, Luis J.; Mora, Marcos G. 1997. Estudio del Mercado de la Carne Bovina. Documento de Trabajo. ODEPA. Chile.

Navarro, Jorge E. 2005. Tópicos de Producción Bovina. Primera Edición., Fundación Chile, Área Agroindustria. Chile.

NAVARro, Jorge E. 2006. El Mercado Mundial de Carnes Bovina y Ovina desde la perspectiva de Chile. Primera Edición., Fundación Chile, Área Agroindustria. Chile.

SÁEZ, L; Leporati, M; Villalobos, P. 2005. Modelo de Simulación para Estimar Costos de Incorporación a PABCO para la Agricultura Familiar Campesina. Software de apoyo a la Incorporación a PABCO. X Congreso de Economistas Agrarios. Noviembre. Temuco. Chile.

VALLE, P. 2001. Evaluación económica de tres sistemas de producción de carne vaca-cría para la Precordillera Andina de la VIII Región. Tesis de Grado. Facultad de Agronomía. Universidad de Concepción. 30 pp.

Velis, HÉCTOR M. 2006. Evolución y Perspectivas. Producción Pecuaria. Chile: Período 2000-2005 y primer semestre 2006. Instituto Nacional de Estadísticas, INE. Chile. 